\title{
A LOWER BOUND FOR THE WEHRL ENTROPY OF QUANTUM SPIN WITH SHARP HIGH-SPIN ASYMPTOTICS
}

\author{
BERNHARD G. BODMANN \\ Department of Physics, Princeton University, Princeton, NJ 08544 \\ bgb@math.princeton.edu
}

\begin{abstract}
A lower bound for the Wehrl entropy of a single quantum spin is derived. The high-spin asymptotics of this bound coincides with Lieb's conjecture up to, but not including, terms of first and higher order in the inverse spin quantum number. The result presented here may be seen as complementary to the verification of the conjecture in cases of lowest spin by Schupp [Commun. Math. Phys. 207 (1999), 481]. The present result for the Wehrl-entropy is obtained from interpolating a sharp norm bound that also implies a sharp lower bound for the socalled Rényi-Wehrl entropy with certain indices that are evenly spaced by half of the inverse spin quantum number.
\end{abstract}

\section{INTRODUCTION}

Among the many facets of entropy, Wehrl Weh91 investigated the relation between the usual Gibbs-Boltzmann-Shannon entropy of a density in phase space and its quantum analogue, commonly known as the von Neumann entropy of a quantum state. The question how to recover one from the other in the sense of a classical limit led Wehrl to construct a quasiclassical entropy of quantum states Weh79. This hybrid construction derived from so-called Glauber coherent states is non-negative, or more accurately, bounded below by the von Neumann entropy. Described in physical terms, the presence of an uncertainty principle in the quasi-classical formulation imposes the lower bound on the usual classical entropy.

Wehrl conjectured that the minimum value of his quasi-classical entropy is assumed whenever the quantum system is in a coherent state. This was proved by Lieb [Lie78] who established sharp norm bounds for the Bargmann transform Bar61, an isometry between Schrödinger's position-space representation and a phase-space representation associated with Glauber coherent states. In addition, Lieb Lie78 suggested that coherent states should also be the minimizers for the Wehrl entropy of quantum spin systems. The quasi-classical entropy for a single quantum spin is derived from coherent states that contain a highest-weight vector in a finite-dimensional Hilbert space carrying a unitary irreducible representation of $\mathrm{SU}(2)$. In this finitedimensional setting, one could have hoped for a simpler way to prove the 
corresponding bound on the Wehrl entropy compared to the effort and ingenuity in the proof given by Lieb for the case of Glauber coherent states. In fact, this would have provided an alternative to Lieb's original proof via the usual group-contraction procedure, in which Glauber coherent states are obtained as the high-spin limit of $\mathrm{SU}(2)$ coherent states [ACGT72. However, despite a lot of attempts, such a proof did not materialize.

Perhaps the simplest proof of Wehrl's original conjecture was given by Carlen Car91 and in a similar argument by Luo Luo00. It reduces to an equality between the norm of functions in the phase-space representation and the Dirichlet form of the Laplacian, together with Gross' logarithmic Sobolev inequality Gro75. Unfortunately, the combination of such identities on the sphere does not yield the desired bound for the Wehrl entropy of a quantum spin. The reason is that the logarithmic Sobolev inequality on the sphere MW82 has the constant as its optimizer, a function that is not contained in the phase-space representation for spin.

A few years ago, Lieb's conjecture was verified for the non-trivial case of spin quantum numbers $j=1$ and $j=3 / 2$ Sch99. This was done by explicit calculation of the entropy in a geometric parametrization of quantum states that also appeared in Lee88, combined with elementary inequalities involving chordal distances of points on the sphere. Here, we derive a lower bound on the Wehrl entropy that coincides with Lieb's conjecture up to first order in the inverse of the spin quantum number. To this end, we modify Carlen's approach and its implicit idea that the Wehrl entropy bound should be a result of the hypercontractivity properties of the Berezin transform. In physical terms, increasing the value of Planck's constant adds fluctuations and smoothes the phase-space density associated with a given quantum state. As usual, we evaluate the smoothness of a density by the $s$-dependence of its $s$-norm, and understand the Wehrl entropy as related to the infinitesimal change at $s=1$. Carlen's technique amounts to establishing bounds between norms for continuously tuned values of Planck's constant. Its analogue in the setting of spin is the inverse of the spin quantum number that cannot be tuned continuously, which is ultimately the reason why the entropy bound obtained here is not sharp. Fortunately, for large spin quantum numbers this conceptual problem is less and less relevant which leads to the sharp asymptotics. A more detailed explanation of the way that Carlen's approach has been modified here is given in the concluding remarks in Section [5.

This paper is organized as follows: In Section 2 we fix the notation and give the basic definitions for a quantum spin. Then, we recall conjectures concerning Wehrl's entropy and some background information in Section 3 . After that, we present a sharp norm bound and derive an estimate on the Wehrl entropy, followed by proofs in Section 4. We conclude with a review of the strategies employed here and possible improvements. 


\section{BASIC DEFINITIONS}

The mathematical description of a spatially fixed, non-relativistic spinning quantum particle involves a $(2 j+1)$-dimensional Hilbert space, where $j \in \frac{1}{2} \mathbb{N}$ is called the spin quantum number. One may identify this space with the complex Euclidean space $\mathbb{C}^{2 j+1}$ equipped with the canonical inner product. The symmetry group describing the purely internal quantum degree of freedom is $\mathrm{SU}(2)$, and its representation on $\mathbb{C}^{2 j+1}$ may be constructed by an inductive procedure Lee88, Sch99] from that on $\mathbb{C}^{2}$. An alternative to this construction is based on choosing a function space related to a coherentstate resolution of the identity Kla59, Ber74, Per86]. This function space incorporates a correspondence principle and makes contact with a classical mechanical system having the sphere as its phase space. In the co-ordinates we use, the functions are defined on the Riemann sphere, i. e. the compactified complex plane. Elements of $\mathrm{SU}(2)$ act in close analogy with rotations, realized as Moebius transformations in the complex plane.

Definition 2.1. Each vector $f$ in the complex linear space $\mathcal{F}_{j}$ is given as the product of a conformal factor and a polynomial of degree at most $2 j$,

$$
f: \mathbb{C} \rightarrow \mathbb{C}, z \mapsto \frac{1}{\left(1+|z|^{2}\right)^{j}} \sum_{k=0}^{2 j} c_{k} z^{k}
$$

with complex coefficients $c_{k} \in \mathbb{C}, k \in\{0,1, \ldots, 2 j\}$. We define an inner product $\langle f, g\rangle$ between vectors $f$ and $g$ as

$$
\langle f, g\rangle:=\frac{2 j+1}{\pi} \int_{\mathbb{C}} \overline{f(z)} g(z) \frac{d^{2} z}{\left(1+|z|^{2}\right)^{2}},
$$

by convention conjugate linear in the first entry.

Remarks 2.2. The elements of the group $\mathrm{SU}(2)$ may be thought of as matrices $(\alpha,-\bar{\beta} ; \beta, \bar{\alpha})$ that are specified by two complex parameters $\alpha, \beta \in \mathbb{C}$ satisfying $|\alpha|^{2}+|\beta|^{2}=1$. The group acts on a function $f$ in the space $\mathcal{F}_{j}$ by Moebius transformations of the argument combined with a unimodular multiplier,

$$
T_{\alpha, \beta} f(z)=\frac{(\beta z+\bar{\alpha})^{2 j}}{|\beta z+\bar{\alpha}|^{2 j}} f\left(\frac{\alpha z-\bar{\beta}}{\beta z+\bar{\alpha}}\right) .
$$

One may verify that indeed the inner product of $\mathcal{F}_{j}$ is invariant under such transformations $\left\{T_{\alpha, \beta}\right\}$, because $\frac{d^{2} z}{\left(1+|z|^{2}\right)^{2}}$ is the rotation-invariant measure on the sphere expressed in stereographic coordinates.

The space $\mathcal{F}_{j}$ is equipped with a reproducing kernel $K$,

$$
K(z, w)=\frac{(1+z \bar{w})^{2 j}}{\left(1+|z|^{2}\right)^{j}\left(1+|w|^{2}\right)^{j}}
$$

that yields

$$
\langle K(\cdot, w), f\rangle=f(w)
$$


for all $f \in \mathcal{F}_{j}, w \in \mathbb{C}$. The functions $\{K(\cdot, w)\} \subset \mathcal{F}_{j}$, indexed by $w \in$ $\mathbb{C} \cup\{\infty\}$ with the convention $K(z, \infty)=\frac{z^{2 j}}{\left(1+|z|^{2}\right)^{j}}$, are also known as coherent vectors.

\section{Conjectured NORM AND ENTROPy BOUndS}

A correspondence principle Ber74 suggests viewing the compactified complex plane as the phase-space of a classical system. From this point of view, the definition of the Hilbert space $\mathcal{F}_{j}$ implies an uncertainty principle. Unlike in $L^{2}$-spaces, one cannot arbitrarily concentrate the contribution to the norm of a function $f \in \mathcal{F}_{j}$ around a given point.

Definition 3.1. The vectors in $\mathcal{F}_{j}$ are by their definition all bounded functions and thus contained in the $L^{p}\left(\mathbb{S}^{2}\right)$ spaces on the Riemann sphere $\mathbb{S}^{2}=\mathbb{C} \cup\{\infty\}$. For each $p \geq 1$, we define the normalized $p$-norm of $f$ as

$$
\|f\|_{p}:=\left(\frac{p j+1}{\pi} \int_{\mathbb{C}}|f(z)|^{p} \frac{d^{2} z}{\left(1+|z|^{2}\right)^{2}}\right)^{1 / p} .
$$

Remarks 3.2. Since the underlying measure is invariant under Moebius transformations, these $p$-norms are all invariant under the action of $\mathrm{SU}(2)$,

$$
\left\|T_{\alpha, \beta} f\right\|_{p}=\|f\|_{p}
$$

where again $\alpha, \beta \in \mathbb{C}$ satisfy $|\alpha|^{2}+|\beta|^{2}=1$.

The unusual, explicit $p$-dependence via the prefactor $p j+1$ in the definition (6) is chosen in order to have $\|K(\cdot, w)\| \|_{p}=1$ independent of $w \in$ $\mathbb{C} \cup\{\infty\}$ and $p \geq 1$. This may be verified by explicit calculation after using an appropriate $T_{\alpha, \beta}$ rotating the index $w$ to the origin. By the point-evaluation property (5), the Cauchy-Schwarz inequality and the normalization of coherent vectors, we estimate $|f(z)| \leq\|f\|_{2}$. Therefore, when $f$ is normalized according to $\|f\|_{2}=1$, the probability density $\rho_{f}: z \mapsto|f(z)|^{2} \leq 1$ cannot be arbitrarily concentrated, which provides a first albeit crude uncertainty principle.

Definition 3.3. The Wehrl entropy of $f \in \mathcal{F}_{j}$, normalized according to $\|f\|_{2}=1$, is defined as

$$
S_{j}\left(|f|^{2}\right):=-\frac{2 j+1}{\pi} \int_{\mathbb{C}}|f(z)|^{2} \ln |f(z)|^{2} \frac{d^{2} z}{\left(1+|z|^{2}\right)^{2}} .
$$

Remark 3.4. By the pointwise estimate in the preceding remark, $S_{j}$ is seen to be non-negative. This may also be proved using Jensen's inequality [Lie78]. Coherent vectors are believed to be the most concentrated wavepackets and therefore expected to minimize $S_{j}$ and various other measures of uncertainty. This is suggestive in the light of the point evaluation property (5), which is in sufficiently large function spaces solely achieved by integrating against Dirac's delta function. 
The somewhat vague notion of coherent vectors being the "most concentrated" motivates the following conjecture in the spirit of Lieb's approach to the Wehrl entropy bound Lie78.

Conjecture 3.5. The normalized $p$-norm of a given $f \in \mathcal{F}_{j}$ is decreasing in $p \geq 1$. More precisely,

$$
\|f \mid\|_{q} \leq\|f\|_{p}
$$

for all $q \geq p \geq 1$, and when $q>p$, equality holds if and only if $f$ is collinear with a coherent vector

$$
f(z)=c K(z, w)
$$

for some fixed pair $c \in \mathbb{C}$ and $w \in \mathbb{C} \cup\{\infty\}$.

A related conjecture has recently been discussed in the literature in the context of measures of localization in phase-space, see Ġ̈01 and references therein. It appears there as a conjectured lower bound for the so-called Rényi-Wehrl entropy. In terms of our notation, it amounts to stating the following special case of the above conjecture.

Conjecture 3.6. Specializing to $p=2$ in the preceding conjecture and using the monotonicity properties of the $q$-th power and of the logarithm, (9) implies for $f \in \mathcal{F}_{j}$ with $\|f\|_{2}=1$ and any $q>2$ that

$$
\frac{2}{2-q} \ln \left(\|f \mid\|_{q}^{q}\right) \geq 0
$$

with equality again if and only if $f$ is collinear with a coherent vector.

We henceforth refer to the left-hand side of the above inequality as an entropy of Rényi-Wehrl type of index $q / 2$, but caution the reader that it differs from the definition of the usual Rényi-Wehrl entropy in the literature GZ̈01, Sug02 by the explicit $q$-dependence contained in the norm $\|f\|_{q}$. A virtue of the normalization used here is that neither (9) nor (11) are explicitly $j$-dependent. In addition, the conjectured monotonicity in (9) and non-negativity in (11) are notationally identical with properties of Rényi's entropy in discrete measure spaces, see Życ03 or equivalent statements in BS93. Section 5.3] in terms of the Rényi information. The reason for this similarity is that the minimizers of Rényi's entropy in the discrete measure spaces are normalized with respect to all $l^{q}$-norms, just as the coherent vectors are in our case. One may wonder whether the counterparts of other properties described in $\dot{\mathrm{Z} y c 03}$ or BS93, such as convexity and monotonicity of $\frac{2}{2-q} \ln \left(\|f\|_{q}^{q}\right)$ in $q$, are also satisfied.

The analogue of (11) has been shown to hold when $q$ is an even integer in a rather general setting Sug02, thereby extending a tensorization argument for SU(2), see [Lee88 or Sch99, Theorem 4.3], to compact semisimple Lie groups, see also the related results [Sai80, Bur83, Bur87, Luo97. However, so far we still lack a proof that includes those $q$ that are arbitrarily close to $p=2$. If those were included in a proof of either of the preceding 
conjectures, then the following bound on the Wehrl entropy would result by endpoint differentiation.

Conjecture 3.7 (Lieb, 1978). For all $f \in \mathcal{F}_{j}$ with $\|f\|_{2}=1$, the Wehrl entropy is bounded below by

$$
S_{j}\left(|f|^{2}\right) \geq \frac{2 j}{2 j+1}
$$

and equality holds whenever $f$ is up to a unimodular constant given by a coherent vector.

\section{Results}

The first result presented in this section is that for a given $f \in \mathcal{F}_{j}$, the norm $\|f\|_{q}$ is decreasing when restricted to a discrete set of values for $q$. A special case of this result implies via interpolation and endpoint differentiation the bound on the Wehrl entropy with sharp asymptotics.

Theorem 4.1. The conjectured norm estimate (9) holds for all $f \in \mathcal{F}_{j}$ if $q=p+\frac{n}{j}$ with any integer $n \in \mathbb{N}$ and $p>\frac{1}{j}$. The estimate is sharp because equality holds in (9) if $f$ is collinear with a coherent vector.

Consequence 4.2. Specializing to $p=2$ and $\|f\|_{2}=1$, this estimate implies via the monotonicity properties of the logarithm and of the $q$-th power that (11) is true for $f \in F_{j}$ when $q=2+\frac{n}{j}$ with $n \in \mathbb{N}$.

Unlike in previous results Sai80, Bur83, Bur87, Luo97, Sch99, Sug02, the index $q / 2$ is no longer restricted to integer values. Another, less direct consequence of Theorem 4.1 is the following bound on Wehrl's entropy.

Theorem 4.3. The Wehrl entropy associated with $f \in \mathcal{F}_{j},\|f\|_{2}=1$, is bounded below by

$$
S_{j}\left(|f|^{2}\right) \geq 2 j \ln \left(1+\frac{1}{2 j+1}\right) .
$$

Remarks 4.4. This bound has the conjectured high-spin asymptotics up to, but not including, first and higher order terms in $j^{-1}$, because

$$
0 \leq \frac{2 j}{2 j-1}-2 j \ln \left(1+\frac{1}{2 j+1}\right)=\frac{2 j}{(2 j+1)^{2}} \int_{0}^{1} \frac{x}{1+\frac{x}{2 j+1}} d x<\frac{1}{4 j} .
$$

Since highest-weight SU(2) coherent states approach Glauber coherent states when scaled appropriately in the limit $j \rightarrow \infty$ [ACGT72, see also [BLW99, one could use these asymptotics to reproduce the entropy bound related to Glauber coherent states obtained by Lieb [Lie78. However, the route via finite dimensional spaces and $\mathrm{SU}(2)$ does not seem to offer the vast simplification that was originally expected.

By the usual concavity argument Weh79, Lie78, Sch99, the Wehrl entropy bound derived here may be extended to include all quantum states, not only pure ones. To this end, we only need to replace the density $\rho_{f}=|f|^{2}$ 
that appears in the definition of $S_{j}$ by the more general $\rho: z \mapsto \sum_{k}\left|f_{k}(z)\right|^{2}$ with any orthogonal family $\left\{f_{n}\right\}_{k=1}^{2 j} \subset \mathcal{F}_{j}$ that satisfies $\sum_{k}\left\|f_{k}\right\|_{2}^{2}=1$. Another possible generalization of the bound (13) is to include several degrees of freedom using the monotonicity of Wehrl's entropy Weh79, Lie78.

\subsection{Proof of Theorem 4.1.}

Proof. To begin with, we remark that it is enough to show the result for $n=1$ and $q>p>q / 2$, otherwise we simply iterate the inequality. The strategy for the proof of Theorem 4.1 is as follows: First, we restate the norm bound (9) as the result of an optimization problem involving the quadratic form of the Laplacian on the sphere. This step relies on identity (23) that is derived in the spirit of Carlen Car91 from the special properties of the function space $\mathcal{F}_{j}$. Then, we enlarge the space when looking for optimizers without losing the sharpness of the bound, because coherent vectors are optimal functions.

For the detailed explanation, it is convenient to introduce the norm $\|f\|_{p}=$ $\|f\|_{p} /(p j+1)^{1 / p}$ of $f \in L^{p}\left(\mathbb{S}^{2}\right)$ with respect to the rotationally-invariant probability measure on $\mathbb{S}^{2}$. Using the Carlen Identity (23), we have for $q \geq p>0$

$$
\max _{f \in \mathcal{F}_{j} \backslash\{0\}} \frac{\|f\|_{q}^{q}}{\|f\|_{p}^{q}}=\max _{f \in \mathcal{F}_{j} \backslash\{0\}} \frac{\|f\|_{q}^{q}-\left.\left.\frac{4}{q j(q j+2)} \int|\partial| f\right|^{q / 2}\right|^{2} \frac{d^{2} z}{\pi}}{\left(1-\frac{1}{q j+2}\right)\|f\|_{p}^{q}} .
$$

Without loss of generality, we can assume that each $f$ vanishes at infinity, $\lim _{z \rightarrow \infty} f(z)=0$. Either this assumption is satisfies right away, or the polynomial part of $f$ has highest degree and thus it has a zero in the complex plane that can be rotated to infinity by a suitable Moebius transformation without changing any of the norms in (15). Now we change notation to $u=|f|^{q / 2}$ and enlarge the set of such $f$,

$$
\max _{f} \frac{\|f\|_{q}^{q}}{\|f\|_{p}^{q}} \leq \sup _{u} \frac{\|u\|_{2}^{2}-\frac{4}{q j(q j+2)} \int|\partial u|^{2} \frac{d^{2} z}{\pi}}{\left(1-\frac{1}{q j+2}\right)\|u\|_{2 p / q}^{2}}
$$

where the supremum is taken over the set $\{0 \leq u \not \equiv 0\}$ in the space $C_{0}^{1}(\mathbb{C})$ of bounded, continuously differentiable functions on the plane that vanish at infinity. In Lemma 4.7 we see that the supremum in (16) has a finite value and even deduce the existence of a maximizing function for this supremum. Given any maximizer, performing a spherically symmetric decreasing rearrangement must necessarily preserve its gradient norm. Therefore, we may restrict ourselves to proving in Lemma 4.9 that in the class of rotationally symmetric functions, this maximizer is unique, up to an overall positive constant. It satisfies the Euler-Lagrange variational equation

$$
\left(1+\frac{4}{q j(q j+2)} \Delta\right) u=b u^{\frac{2 p}{q}-1}
$$


with $\Delta=\frac{1}{4}\left(1+|z|^{2}\right)^{2}\left(\partial_{1}^{2}+\partial_{2}^{2}\right)$ being the spherical Laplacian and a constant $b>0$ that is fixed by choosing the norm $\|u\|_{2 p / q}$. By inspection, this unique function is seen to be $u: z \mapsto A\left(1+|z|^{2}\right)^{q j / 2}$ with $A=((q j+2) b / q j)^{q / 2(q-p)}$, and thus the corresponding $f$ is up to a constant factor a coherent vector.

Consequently, inequality (9) follows from reverting to the original normalization convention. It is sharp since equality is achieved if $f$ is a coherent vector.

4.2. Proof of Theorem 4.3. This is merely a consequence of Theorem 4.1. It follows from interpolating the norm bound (9) and endpoint differentiation.

Proof. Let us choose $q=2+\frac{1}{j}$ in Theorem 4.1 and for $2<s<q$ select $\theta>0$ such that $\frac{1}{s}=\frac{1-\theta}{2}+\frac{\theta}{q}$. Using the same notation $\|f\|_{s}=\|f\|_{s} /(s j+1)^{1 / s}$ as before, Hölder's inequality combined with the norm bound (9) gives

$$
\begin{aligned}
\|f\|_{s} & \leq\|f\|_{2}^{1-\theta}\|f\|_{q}^{\theta} \\
& \leq\left[\frac{(2 j+1)^{1 / 2}}{(q j+1)^{1 / q}}\right]^{\theta}\|f\|_{2},
\end{aligned}
$$

so eliminating $\theta$ results in

$$
\|f\|_{s}^{s} \leq \frac{(2 j+1)^{\frac{q}{2} \frac{2-s}{2-q}}}{(q j+1)^{\frac{2-s}{2-q}}}\|f\|_{2}^{s} .
$$

At $s=2$ both sides are equal, thus one may differentiate

$$
\begin{aligned}
& \left.s \frac{d}{d s}\right|_{s=2}\|f\|_{s}^{s}=\frac{1}{\pi} \int_{\mathbb{C}}|f(z)|^{2} \ln |f(z)|^{2} \frac{d^{2} z}{\left(1+|z|^{2}\right)^{2}} \\
& \leq\left. s \frac{d}{d s}\right|_{s=2} \frac{(2 j+1)^{\frac{q}{2} \frac{2-s}{2-q}}}{(q j+1)^{\frac{2-s}{2-q}}}=\ln (2 j+1)-2 j \ln \left(\frac{2 j+2}{2 j+1}\right)
\end{aligned}
$$

and by a change in normalization and an overall change of sign arrives at the desired entropy bound (13). The justification for differentiating under the integral sign in (21) is dominated convergence and the estimate $0 \geq$ $2\left(|f|^{s-2}-1\right) /(s-2) \geq \ln |f|^{2}$.

\subsection{Ingredients of the proof of Theorem 4.1.}

Lemma 4.5 (Carlen Identity). For $q>0$ and all $f \in \mathcal{F}_{j}, j \in \frac{1}{2} \mathbb{N}$, the following identity is true

$$
\left.\left.\int_{\mathbb{C}}|\partial| f\right|^{q / 2}\right|^{2} \frac{d^{2} z}{\pi}=\frac{q j}{4 \pi} \int_{\mathbb{C}}|f|^{q} \frac{d^{2} z}{\left(1+|z|^{2}\right)^{2}} .
$$


Proof. We will first prove a regularized version of the identity. Let $\epsilon>0$ and assume $\phi: \mathbb{C} \rightarrow \mathbb{C}$ is a complex polynomial of maximal degree $2 j$, we will show for $q>0$ that

$$
\int_{\mathbb{C}}\left|\partial \frac{\left(|\phi(z)|^{2}+\epsilon\right)^{q / 4}}{\left(1+|z|^{2}\right)^{q j / 2}}\right|^{2} \frac{d^{2} z}{\pi}=\frac{q j}{4} \int_{\mathbb{C}} \frac{\left(|\phi(z)|^{2}+\epsilon\right)^{q / 2}}{\left(1+|z|^{2}\right)^{q j+2}} \frac{d^{2} z}{\pi}+E(\epsilon)
$$

with an error term

$$
E(\epsilon)=\frac{\epsilon q}{8} \int_{\mathbb{C}}\left(|\phi(z)|^{2}+\epsilon\right)^{\frac{q}{2}-2} \frac{|\partial \phi(z)|^{2}}{\left(1+|z|^{2}\right)^{q j}} \frac{d^{2} z}{\pi}
$$

This regularized identity is obtained by elementary calculus operations involving the complex derivative $\partial$, observing that $\phi$ is holomorphic, and integration by parts:

$$
\begin{aligned}
& \int_{\mathbb{C}}\left|\partial \frac{\left(|\phi(z)|^{2}+\epsilon\right)^{q / 4}}{\left(1+|z|^{2}\right)^{q j / 2}}\right|^{2} \frac{d^{2} z}{\pi}=\int_{\mathbb{C}}\left[\frac{q^{2}}{16} \frac{|\phi(z)|^{2}|\partial \phi(z)|^{2}\left(|\phi(z)|^{2}+\epsilon\right)^{\frac{q}{2}-2}}{\left(1+|z|^{2}\right)^{q j}}\right. \\
& \left.-\frac{q^{2} j}{4} \frac{\Re[\overline{\phi(z)} z \partial \phi(z)]\left(|\phi(z)|^{2}+\epsilon\right)^{\frac{q}{2}-1}}{\left(1+|z|^{2}\right)^{q j+1}}+\frac{q^{2} j^{2}}{4} \frac{|z|^{2}\left(|\phi(z)|^{2}+\epsilon\right)^{q j / 2}}{\left(1+|z|^{2}\right)^{q j+2}}\right] \frac{d^{2} z}{\pi} \\
& =\int_{\mathbb{C}}\left[\frac{1}{\left(1+|z|^{2}\right)^{q j}}\left(\frac{1}{4} \partial \bar{\partial}\left(|\phi(z)|^{2}+\epsilon\right)^{q / 2}+\epsilon \frac{q}{8}\left(|\phi(z)|^{2}+\epsilon\right)^{\frac{q}{2}-2}|\partial \phi(z)|^{2}\right)\right. \\
& \left.-\frac{q j}{2} \frac{\Re\left[z \partial\left(|\phi(z)|^{2}+\epsilon\right)^{q / 2}\right]}{\left(1+|z|^{2}\right)^{q j+1}}+\frac{q^{2} j^{2}}{4} \frac{|z|^{2}\left(|\phi(z)|^{2}+\epsilon\right)^{q / 2}}{\left(1+|z|^{2}\right)^{q j+2}}\right] \frac{d^{2} z}{\pi} \\
& =\int_{\mathbb{C}}\left(|\phi(z)|^{2}+\epsilon\right)^{q / 2}\left[\frac{1}{4} \partial \bar{\partial} \frac{1}{\left(1+|z|^{2}\right)^{q j}}+\epsilon \frac{q}{8} \frac{|\partial \phi(z)|^{2}}{\left(|\phi(z)|^{2}+\epsilon\right)^{2}}\right. \\
& \left.-\frac{q j}{2} \Re\left[\partial \frac{z}{\left(1+|z|^{2}\right)^{q j+1}}\right]+\frac{q^{2} j^{2}}{4} \frac{|z|^{2}}{\left(1+|z|^{2}\right)^{q j+2}}\right] \frac{d^{2} z}{\pi} \\
& =\frac{q j}{4} \int_{\mathbb{C}} \frac{\left(|\phi(z)|^{2}+\epsilon\right)^{q / 2}}{\left(1+|z|^{2}\right)^{q j+2}} \frac{d^{2} z}{\pi}+\epsilon \frac{q}{8} \int_{\mathbb{C}} \frac{\left(|\phi(z)|^{2}+\epsilon\right)^{\frac{q}{2}-2}|\partial \phi(z)|^{2}}{\left(1+|z|^{2}\right)^{q j}} \frac{d^{2} z}{\pi} .
\end{aligned}
$$

There are no boundary terms in the integration by parts because the growth of the powers of $|\phi|$ and of their derivatives are suppressed by sufficiently strong polynomial growth in the denominator.

In the limit $\epsilon \rightarrow 0$, the error term vanishes by dominated convergence, yielding identity (23). Dominated convergence applies because either $q \geq$ 4 and $\left(|\phi(z)|^{2}+\epsilon\right)^{\frac{q}{2}-2} \leq C\left(1+|z|^{2}\right)^{j(q-4)}$ with a fixed constant $C>0$ valid for all sufficiently small $\epsilon$, or $0<q<4$ and we estimate on the set $\left\{z:|\phi(z)|^{2}<\epsilon\right\}$ the expression $\epsilon\left(|\phi(z)|^{2}+\epsilon\right)^{\frac{q}{2}-2} \leq 2 \frac{(4-q)^{2-\frac{q}{2}}}{(2-q)^{3-\frac{q}{2}}}|\phi(z)|^{q-2}$. To ensure the validity of dominated convergence, one may then recall that $\phi$ has only isolated zeros of finite order. 
Remark 4.6. A version of the identity (23) was first shown by Carlen Car91 for the function space related to Glauber coherent states. One may recover his result by the usual group contraction procedure in the limit $j \rightarrow \infty$ while scaling $z \rightarrow z / \sqrt{2 j \hbar}$ with $\hbar>0$.

The case $q=2$ can be verified in a simpler way than the explicit calculation given here. All that is needed is to verify that the square of the gradient norm in (23) defines a quadratic form on $\mathcal{F}_{j}$. Appealing to Schur's lemma, the corresponding operator is a multiple of the identity, because the quadratic form is invariant under the irreducible unitary representation of $\mathrm{SU}(2)$. To obtain the correct numerical factor, one may then simply choose $f$ to be the coherent vector centered at the origin and evaluate the left-hand side of equation (23).

Lemma 4.7. The supremum of the expression (16) is attained for some function $u$. In other words, there is some $u$ with $\|u\|_{s}=1,1<s<2$, satisfying $0 \leq u$ and $u(z) \rightarrow 0$ as $z \rightarrow \infty$, which maximizes

$$
\|u\|_{2}^{2}-\frac{4}{q j(q j+2)} \int_{\mathbb{C}}|\partial u|^{2} \frac{d^{2} z}{\pi} .
$$

Proof. We abbreviate $s:=\frac{2 p}{q}$ and denote the dual index as $s^{\prime}:=\frac{s}{s-1}$. The Sobolev inequality on the two-sphere states

$$
\|u\|_{2}^{2}+\frac{s^{\prime}-2}{2}\|\nabla u\|_{2}^{2} \geq\|u\|_{s^{\prime}}^{2} .
$$

Note that for real-valued $u$, in two dimensions the two expressions $\|\nabla u\|_{2}^{2}$ and $\int_{\mathbb{C}}|\partial u|^{2} d^{2} z / \pi$ are identical, one using the gradient $\nabla$ on the sphere and the other the complex derivative $\partial=\left(\partial_{1}-i \partial_{2}\right) / 2$. Therefore, employing the Sobolev and Hölder inequalities yields for $\|u\|_{s}=1$ a finite bound

$$
\begin{aligned}
& \|u\|_{2}^{2}-\frac{4}{q j(q j+2)} \int_{\mathbb{C}}|\partial u|^{2} \frac{d^{2} z}{\pi} \\
& \leq\left(1+\frac{2}{r-2} \frac{4}{q j(q j+2)}\right)\|u\|_{s}\|u\|_{s^{\prime}}-\frac{2}{r-2} \frac{4}{q j(q j+2)}\|u\|_{s^{\prime}}^{2} \\
& \leq \max _{x \geq 0}\left[\left(1+\frac{2}{r-2} \frac{4}{q j(q j+2)}\right) x-\frac{2}{r-2} \frac{4}{q j(q j+2)} x^{2}\right] .
\end{aligned}
$$

Now assume a maximizing sequence $\left(u_{n}\right)_{n \in \mathbb{N}}$ such that $0 \leq u_{n}$ and $\left\|u_{n}\right\|_{s}=$ 1 for all $n \in \mathbb{N}$. Apart from the finiteness of the supremum over $s$-normalized $u$ in (32), inequality (33) shows that the sequence $\left(x_{n}\right)_{n \in \mathbb{N}}$ given by $x_{n}:=$ $\left\|u_{n}\right\|_{s^{\prime}}$ is bounded, and because of the compactness of the sphere, the same bound applies to the 2-norms of the sequence $\left(u_{n}\right)_{n \in \mathbb{N}}$. This, in turn, forces any maximizing sequence to be uniformly bounded in gradient 2-norm.

We may now without loss of generality replace each $u_{n}$ by its equimeasurable symmetric decreasing rearrangement, since this only decreases the gradient norm by the contractivity properties of the heat semigroup on the sphere, just as in the Euclidean case Lie83, Lemma 4.1]. In addition, we 
can use Helly's selection principle, that is, choose a subsequence that converges on all rational radii. By the monotonicity of each $u_{n}$, the convergence extends to almost every radius. Appealing to a Rellich-Kondrakov compact embedding theorem Aub82, Theorem 2.34], the limit $u_{n} \rightarrow u$ is in the $s$ norm. The compact embedding we use is that of the Sobolev space $H_{1}^{1}\left(\mathbb{S}^{2}\right)$ in $L^{s}\left(\mathbb{S}^{2}\right)$.

Lemma 4.8. Given $s>1$, every maximizer $u \in L^{s}\left(\mathbb{S}^{2}\right)$ in the preceding lemma is a smooth function.

Proof. From calculus of variations, any maximizer $u \in L^{s}\left(\mathbb{S}^{2}\right)$ is a distributional solution of the corresponding Euler-Lagrange equation that has the form of a Poisson equation,

$$
\Delta u=a u^{s-1}-b u
$$

with constants $a, b>0$. By the integrability of the Green function $G$ on the sphere, in complex coordinates given as

$$
G(z, w)=-\ln \left(\frac{4|z-w|^{2}}{\left(1+|z|^{2}\right)\left(1+|w|^{2}\right)}\right),
$$

and the identity

$$
u(w)=\frac{1}{\pi} \int_{\mathbb{C}} G(w, z)\left(a u^{s-1}(z)-b u(z)\right) \frac{d^{2} z}{\left(1+|z|^{2}\right)^{2}}
$$

we see that $u$ is bounded and continuous.

In fact, its first derivative is seen to be Hölder continuous with any index $0<\alpha<1$ from replacing $u$ with a directional derivative and calculating the magnitude of the gradient of the Green function

$$
|\nabla G(z, w)|=\left(\frac{\left(1+|z|^{2}\right)\left(1+|w|^{2}\right)}{|z-w|^{2}}-\frac{1}{\left(1+|z|^{2}\right)\left(1+|w|^{2}\right)}\right)^{1 / 2} \text {. }
$$

By bootstrapping, $u$ is smooth since its $k$-th derivative is Hölder continuous with index $0<\alpha_{k}<(s-1)^{k-1}$.

Lemma 4.9. Given $q>p>q / 2$, there is a unique rotationally symmetric non-trivial solution $u$ to the Euler-Lagrange equation (17) that meets the non-negativity and limit requirements $u \geq 0$ and $\lim _{z \rightarrow \infty} u(z)=0$.

Proof. We know from the preceding lemma that $u$ is differentiable, even smooth. Therefore, a rotationally symmetric $u$ considered as a function of $r=|z|$ solves the ordinary differential equation

$$
\left(1+r^{2}\right)^{2} \frac{1}{r}\left(r u^{\prime}(r)\right)^{\prime}-a(u(r))^{\frac{2 p}{q}-1}+b u(r)=0
$$

with an initial condition $u^{\prime}(0)=0$ and an unknown value $u(0)>0$. We will abbreviate the non-linearity as $\phi(u):=-b u^{(2 p-q) / q}+u$. Despite the singularity at $r=0$, the initial values $u(0)$ and $u^{\prime}(0)=0$ uniquely determine 
a solution on all $\{r \geq 0\}$. This follows with the help of the Schauder fixed point theorem as in [FLS96. Appendix] by replacing the expression for $u^{\prime}(r)$ in the Euclidean case treated there with (39) given hereafter. To show the claimed uniqueness of a solution meeting the non-negativity and limiting requirements, we first show that any such solution is strictly radially decreasing. Then, we exclude the cases of multiple solutions having infinitely many, finitely many, and finally having no intersections. In the literature, such arguments have been called separation lemmas. We adapt elements of PS83, PS86, FLS96, ST00] to the setting of the sphere.

To begin with, we show that $u$ is strictly decreasing as a function of $r$. Integrating the ODE (38) gives

$$
u^{\prime}(r)=-\frac{1}{r} \int_{0}^{r} \frac{t}{\left(1+t^{2}\right)^{2}} \phi(u(t)) d t .
$$

If there some critical point $r_{0}>0$ for $u$, then we could conclude that both sides of this equation vanish and thus, inserting (39) simplifies (38) to

$$
u^{\prime \prime}\left(r_{0}\right)=-\frac{1}{\left(1+r_{0}^{2}\right)^{2}} \phi\left(u\left(r_{0}\right)\right)=0 .
$$

From the uniqueness of solutions to initial value problems having Lipschitzcontinuous coefficients we conclude that $u$ is necessarily the non-zero constant determined by $\phi(u)=0$, contradicting the limiting requirement in our assumption. Therefore, $u$ cannot have any critical points for $r>0$.

Now we exclude the three possible cases of multiple solutions.

(1) Two solutions with infinitely many intersections. Assuming there are two solutions $u$ and $v$ that intersect infinitely often, then there are radii $0<r_{0}<r_{1}$ such that

(a) $u\left(r_{1}\right)=v\left(r_{1}\right), u^{\prime}\left(r_{0}\right)=v^{\prime}\left(r_{0}\right)$, and $0>v^{\prime}>u^{\prime}$ on $\left(r_{0}, r_{1}\right)$,

(b) $\phi(u(r))<0$ and $\phi(v(r))<0$ for all $r \geq r_{0}$.

Define $\Phi(u):=\int_{0}^{u} \phi(x) d x$. By comparison with energy dissipation in a mechanical system, we have the "conservation law", here in terms of the solution $u$,

$$
\begin{aligned}
& {\left[\frac{1}{2}\left(1+r^{2}\right)^{2}\left(u^{\prime}(r)\right)^{2}+\Phi(u(r))\right]_{r_{0}}^{r_{1}}} \\
& \quad+\int_{r_{0}}^{r_{1}}\left(\frac{\left(1+r^{2}\right)^{2}}{r}-r\left(1+r^{2}\right)\right)\left(u^{\prime}(r)\right)^{2} d r=0 .
\end{aligned}
$$

Subtracting this identity for the two solutions $u$ and $v$, we obtain

$$
\begin{aligned}
& \frac{1}{2}\left(1+r_{1}^{2}\right)^{2}\left(\left(u^{\prime}\left(r_{1}\right)\right)^{2}-\left(v^{\prime}\left(r_{1}\right)\right)^{2}\right)+\Phi\left(v\left(r_{0}\right)\right)-\Phi\left(u\left(r_{0}\right)\right) \\
& +\int_{r_{0}}^{r_{1}}\left(\frac{\left(1+r^{2}\right)^{2}}{r}-r\left(1+r^{2}\right)\right)\left(\left(u^{\prime}(r)\right)^{2}-\left(v^{\prime}(r)\right)^{2}\right) d r=0
\end{aligned}
$$

but the left-hand side is strictly positive in each difference term, so this yields a contradiction. 
(2) Two solutions having finitely many intersections. For this part, we choose the parametrization in terms of the azimuthal angle $\theta, r=$ $\tan \theta / 2$. In this variable, the ordinary differential equation (38) is expressed as

$$
u^{\prime \prime}(\theta)+\cot \theta u^{\prime}(\theta)+\phi(u(\theta))=0 .
$$

From the two solutions $u$ and $v$ let us pass to the inverse functions denoted as $\theta=u^{-1}$ and $\sigma=v^{-1}$. If there are finitely many intersections, then there is $u_{0}>0$ such that $\theta>\sigma>\pi / 2$ and $0>\theta^{\prime}>\sigma^{\prime}$ on $\left(0, u_{0}\right)$.

Define the difference of "kinetic energy" as

$$
B(u)=\frac{1}{\left(\theta^{\prime}(u)\right)^{2}}-\frac{1}{\left(\sigma^{\prime}(u)\right)^{2}}>0
$$

then $B(0)=0$. However, according to the ODE (43), the derivative

$$
B^{\prime}(u)=-2\left(\frac{\cot \theta}{\theta^{\prime}}-\frac{\cot \sigma}{\sigma^{\prime}}\right)<0
$$

since $0>\cot \theta>\cot \sigma$ and $0>\theta^{\prime}>\sigma^{\prime}$. We have reached a contradiction with the strict positivity of $B$.

(3) Two solutions without intersection. As a first step to exclude this case, we will show that the difference $\theta-\sigma$ of the inverse functions of $u$ and $v$ can have at most one critical point, a local maximum, in an interval where $\theta>\sigma$.

To this end, we note that in terms of the inverse function $\theta$ of $u$, the ODE (43) becomes

$$
-\theta^{\prime \prime}+\cot \theta\left(\theta^{\prime}\right)^{2}+\phi(u(\theta))\left(\theta^{\prime}\right)^{3}=0 .
$$

Subtracting this identity for the two solutions at a point where $\theta^{\prime}=$ $\sigma^{\prime}$ and $\theta>\sigma$ gives

$$
\theta^{\prime \prime}-\sigma^{\prime \prime}=(\cot \theta-\cot \sigma)\left(\theta^{\prime}\right)^{2}<0
$$

by the monotonicity of the cotangent function.

Consequently, assuming $u>v$, then the difference $\theta-\sigma$ cannot have any critical point in $(0, v(0))$, because this would contradict $\theta^{\prime}-\sigma^{\prime} \rightarrow \infty$ as $u \rightarrow v(0)$. So $\theta^{\prime}-\sigma^{\prime}>0$ in $(0, v(0))$. But similarly as in the preceding case of finitely many intersections, this is impossible near $u=0$, because in terms of the azimuthal angle we would then have $B(0)=0, B^{\prime}(u)<0$ for small $u$.

\section{Conclusion}

The lack of a sharp entropy bound remains frustrating. Many promising attempts have failed. Among others, this includes the hope that grouptheoretic, explicit computations as in Sch99] may give rise to an inductive argument; the use of the Hardy-Littlewood-Sobolev inequality on the sphere 
that may be found in so many uncertainty principles [Bec93, Bec95, Bec01]; or via a change of weight analogous to Luo00 the attempt to combine variants of the Carlen identity with a logarithmic Sobolev-type inequality on the sphere that breaks down because of a domain problem Gro99. Section 5]. At this point the best bet may still be the difficult task of finding sharp smoothing properties of the Berezin transform. For lack of a manageable alternative, we replaced the Berezin transform with the differential operator $1+\frac{4}{q j(q j+2)} \Delta$, which should intuitively be a good approximation for high spin quantum numbers. This particular choice of approximation is motivated by considering optimization problems of the form (16) and by demanding that coherent vectors solve the corresponding Euler-Lagrange equation. The downside of replacing the Berezin transform is that we cannot characterize the cases of equality in Theorem 4.1 because there is no strong form of the Riesz theorem for the gradient norm under radially symmetric decreasing rearrangements. We cannot resolve this deficiency by invoking a duality principle as in [Bec01, Theorem 6], because the operator $1+\frac{4}{q j(q j+2)} \Delta$ is not invertible.

One may expect that an adaptation of the techniques employed here yields analogous bounds for the Wehrl entropy in the setting of $\mathrm{SU}(1,1)$ as well, see [Luo97]. It could be worthwhile to include other highest-weight representations Ġं01. However, the most important task is still to find the road that will finally lead to the sharp bounds. Most likely it will not be a simple group-theoretic argument, but all of the partial results so far point to the very geometric nature and inherent beauty of the problem. Discovering these facets of entropy makes the difficulties in resolving Lieb's conjecture much less frustrating and all the more fascinating.

Acknowledgments. Thanks go to Elliott Lieb for remarks that were always to the point and for suggesting this incredibly beautiful and challenging open problem to me. I am also grateful for helpful and motivating conversations with Shannon Starr, Michael Aizenman, Robert Seiringer, Almut Burchard, Stephen B. Sontz and Bob Sims. The referee is acknowledged for pointing out analogies between the material presented here and classical results for the Rényi entropy on discrete measure spaces. This work was partially supported under the National Science Foundation grant PHY-0139984.

\section{REFERENCES}

[ACGT72] F. T. Arecchi, E. Courtens, R. Gilmore, and H. Thomas, Atomic coherent states in quantum optics, Phys. Rev. A 6 (1972), 2211-2237.

[Aub82] Th. Aubin, Nonlinear analysis on manifolds. Monge-Ampère equations, Grundlehren der mathematischen Wissenschaften, vol. 252, Springer-Verlag, New York, 1982.

[Bar61] V. Bargmann, On a Hilbert space of analytic functions and an associated integral transform, Part I, Comm. Pure Appl. Math. 14 (1961), 187-214.

[Bec93] W. Beckner, Sharp Sobolev inequalities on the sphere and the Moser-Trudinger inequality, Ann. of Math. (2) 138 (1993), no. 1, 213-242. 
[Bec95] , Pitt's inequality and the uncertainty principle, Proc. Amer. Math. Soc. 123 (1995), no. 6, 1897-1905.

[Bec01] On the Grushin operator and hyperbolic symmetry, Proc. Amer. Math. Soc. 129 (2001), no. 4, 1233-1246.

[Ber74] F. A. Berezin, Quantization, Math. USSR Izvestija 8 (1974), 1109-1165, russ. orig.: Izv. Akad. Nauk SSSR, Ser. Mat. 38 (1974), 1116-1175.

[BLW99] B. Bodmann, H. Leschke, and S. Warzel, A rigorous path integral for quantum spin using flat-space Wiener regularization, J. Math. Phys. 40 (1999), 25492559.

[BS93] Ch. Beck and F. Schlögl, Thermodynamics of chaotic systems, Cambridge Nonlinear Science Series, vol. 4, Cambridge University Press, Cambridge, 1993.

[Bur83] J. Burbea, Inequalities for holomorphic functions of several complex variables, Trans. Amer. Math. Soc. 276 (1983), no. 1, 247-266.

[Bur87] _ Sharp inequalities for holomorphic functions, Illinois J. Math. 31 (1987), no. 2, 248-264.

[Car91] E. A. Carlen, Some integral identities and inequalities for entire functions and their application to the coherent state transform, J. Funct. Anal. 97 (1991), no. 1, 231-249.

[FLS96] B. Franchi, E. Lanconelli, and J. Serrin, Existence and uniqueness of nonnegative solutions of quasilinear equations in $\mathbf{R}^{n}$, Adv. Math. 118 (1996), no. 2, 177-243.

[Gro75] L. Gross, Logarithmic Sobolev inequalities, Amer. J. Math. 97 (1975), no. 4, 1061-1083.

[Gro99] _ Hypercontractivity over complex manifolds, Acta Math. 182 (1999), no. 2, 159-206.

[GŻ01] S. Gnutzmann and K. Życzkowski, Rényi-Wehrl entropies as measures of localization in phase space, J. Phys. A 34 (2001), no. 47, 10123-10139.

[Kla59] J. R. Klauder, The action option and a Feynman quantization of spinor fields in terms of ordinary c-numbers., Ph.D. thesis, Princeton, 1959.

[Lee88] C. T. Lee, Wehrl's entropy of spin states and Lieb's conjecture, J. Phys. A 21 (1988), no. 19, 3749-3761.

[Lie78] E. H. Lieb, Proof of an entropy conjecture of Wehrl, Comm. Math. Phys. 62 (1978), no. 1, 35-41.

[Lie83] E. H. Lieb, Sharp constants in the Hardy-Littlewood-Sobolev and related inequalities, Ann. of Math. (2) 118 (1983), no. 2, 349-374.

[Luo97] S. Luo, A harmonic oscillator on the Poincaré disc and hypercontractivity, J. Phys. A 30 (1997), no. 14, 5133-5139.

[Luo00] A A simple proof of Wehrl's conjecture on entropy, J. Phys. A 33 (2000), no. 16, 3093-3096.

[MW82] C. E. Mueller and F. B. Weissler, Hypercontractivity for the heat semigroup for ultraspherical polynomials and on the n-sphere, J. Funct. Anal. 48 (1982), no. 2, 252-283.

[Per86] A. Perelomov, Generalized coherent states and their applications, Texts and Monographs in Physics, Springer-Verlag, Berlin, 1986.

[PS83] L. A. Peletier and J. Serrin, Uniqueness of positive solutions of semilinear equations in $\mathbf{R}^{n}$, Arch. Rational Mech. Anal. 81 (1983), no. 2, 181-197.

[PS86] _ Uniqueness of nonnegative solutions of semilinear equations in $\mathbf{R}^{n}, \mathrm{~J}$. Differential Equations 61 (1986), no. 3, 380-397.

[Sai80] S. Saitoh, Some inequalities for entire functions, Proc. Amer. Math. Soc. 80 (1980), no. 2, 254-258.

[Sch99] P. Schupp, On Lieb's conjecture for the Wehrl entropy of Bloch coherent states, Comm. Math. Phys. 207 (1999), no. 2, 481-493. 
[ST00] J. Serrin and M. Tang, Uniqueness of ground states for quasilinear elliptic equations, Indiana Univ. Math. J. 49 (2000), no. 3, 897-923.

[Sug02] A. Sugita, Proof of the generalized Lieb-Wehrl conjecture for integer indices larger than one, J. Phys. A 35 (2002), no. 42, L621-L626.

[Weh79] A. Wehrl, On the relation between classical and quantum-mechanical entropy, Rep. Math. Phys. 16 (1979), no. 3, 353-358.

[Weh91] The many facets of entropy, Rep. Math. Phys. 30 (1991), no. 1, 119129 (1992).

[Życ03] K. Życzkowski, Rényi extrapolation of Shannon entropy, Open Syst. Inf. Dyn. 10 (2003), no. 3, 297-310. 